\title{
Light-driven permanent transition from insulator to conductor
}

\author{
D. Rana $\odot,{ }^{1}$ S. Agarwal,,${ }^{1,2}$ M. Islam, ${ }^{1,2}$ A. Banerjee, ${ }^{3}$ B. P. Uberuaga $\odot,{ }^{3,}{ }^{*}$ P. Saadatkia,,${ }^{1,2}$ P. Dulal, ${ }^{1}$ \\ N. Adhikari $\odot,{ }^{1}$ M. Butterling, ${ }^{4}$ M. O. Liedke $\odot,{ }^{4}$ A. Wagner $\odot,{ }^{4}$ and F. A. Selim $\odot^{1,2, *}$ \\ ${ }^{1}$ Department of Physics and Astronomy, Bowling Green State University, Bowling Green, Ohio 43403, USA \\ ${ }^{2}$ Center for Photochemical Sciences, Bowling Green State University, Bowling Green, Ohio 43403, USA \\ ${ }^{3}$ Materials Science and Technology Division, Los Alamos National Laboratory, Los Alamos, New Mexico 87545, USA \\ ${ }^{4}$ Institute of Radiation Physics, Helmholtz-Zentrum Dresden-Rossendorf, Bautzner Landstrasse 400, 01328 Dresden, Germany
}

(Received 12 April 2021; revised 9 November 2021; accepted 3 December 2021; published 22 December 2021)

\begin{abstract}
The transition from insulator to conductor can be realized in some materials but requires modification of both the arrangement of atoms and their electronic configurations. This is often achieved by doping. Here we reveal a different mechanism the lattice may adopt to induce such a transition. Experiments showed the surprising finding that limited exposure to subband-gap light caused a permanent transition from an insulator state to a conductor state in the insulating oxide $\mathrm{Ga}_{2} \mathrm{O}_{3}$, with a nine orders of magnitude increase in electronic conduction. Furthermore, annealing up to $400{ }^{\circ} \mathrm{C}$ did not suppress or decrease the induced conductivity. Photoexcitation by light-induced modification in the charge state of defects and subsequent lattice distortion around them was suggested to be the underlying mechanism behind this transition. Density functional theory calculations confirmed that modifying the charge state of defects leads to redistribution of the localized electrons and massive structural distortion in the surrounding lattice, causing large shifts in the density of states and introducing new states with shallower energy levels. Both experimental and theoretical results revealed the introduction of stable shallow energy levels, explaining the mechanism behind the transition from an insulator to a conductor state by light. We suggest that this mechanism may occur in other wide band-gap metal oxides leading to drastic modification in their electronic properties.
\end{abstract}

DOI: 10.1103/PhysRevB.104.245208

\section{INTRODUCTION}

When light is impinged on a semiconductor material, charge carriers-electrons and holes-may be generated resulting in an enhancement in conductivity. If the energy of the incident photons is greater than the band gap of the semiconductor, it excites an electron from the valance band to the conduction band, a phenomenon called intrinsic photoconductivity. On the other hand, if the energy of the photon is less than the band gap, it may excite electrons from defect levels to the conduction band enhancing conductivity. This is referred to as extrinsic photoconductivity [1]. In either case, if the conductivity persists after turning off the photoexcitation, it is known as persistent photoconductivity. In this situation, when the electron-hole pairs are generated, there must be microscopic or macroscopic potential barriers that separate charge carriers and reduce the probability of recombination between them, resulting in enhanced conductivity for a longer period of time [2]. Persistent photoconductivity at room temperature has been primarily reported in heterostructures of semiconductors and in a few bulk materials $[2,3]$.

\footnotetext{
*Corresponding authors: blas@lanl.gov; faselim@bgsu.edu
}

Published by the American Physical Society under the terms of the Creative Commons Attribution 4.0 International license. Further distribution of this work must maintain attribution to the author(s) and the published article's title, journal citation, and DOI.
In this work we report an extrinsic persistent photoconductivity behavior in bulk $\mathrm{Ga}_{2} \mathrm{O}_{3}$ followed by a surprising permanent transition from the insulator state to the conductor state upon exposure to subband-gap light for a limited period of time. First, $\mathrm{Ga}_{2} \mathrm{O}_{3}$ bulk crystals exhibited photoconductivity at room temperature upon exposure to subband-gap light of much lower energy than the band gap that persists for a brief time after turning off the light. Then, by repeated photoexcitation and increasing photoexcitation time, the induced metastable states created by light became stable, leading to a permanent transition from insulator to conductor state. That such a transition can occur just by exposing the sample to light while keeping it at room temperature without any further treatment is surprising and could have vast implications on the material properties and applications.

Gallium oxide $\left(\mathrm{Ga}_{2} \mathrm{O}_{3}\right)$ is the widest band-gap transparent (up to UV-C range) semiconducting oxide known so far [4-25]. Its ultrawide band gap $(\sim 4.5-4.9 \mathrm{eV})$ [6] may lead to unusual electronic phenomena. Due to this wide band gap, UV-C transparency, and excellent thermal and chemical stability, it has numerous potential applications in power and high voltage devices, Schottky diodes, field effect transistors, gas sensors, phosphors and electroluminescent devices, UV photodetectors, and more [7-11,22-24]. $\mathrm{Ga}_{2} \mathrm{O}_{3}$ exhibits polymorphism, denoted by $\alpha, \beta, \gamma, \delta$, and $\varepsilon$ [12], with $\beta$ $\mathrm{Ga}_{2} \mathrm{O}_{3}$ being the most stable phase from room temperature to its melting point [12]. As the most stable form, $\beta-\mathrm{Ga}_{2} \mathrm{O}_{3}$ is also the most studied polymorph. It crystalizes into a monoclinic structure with space group $C 2 / m$ and lattice parameters 

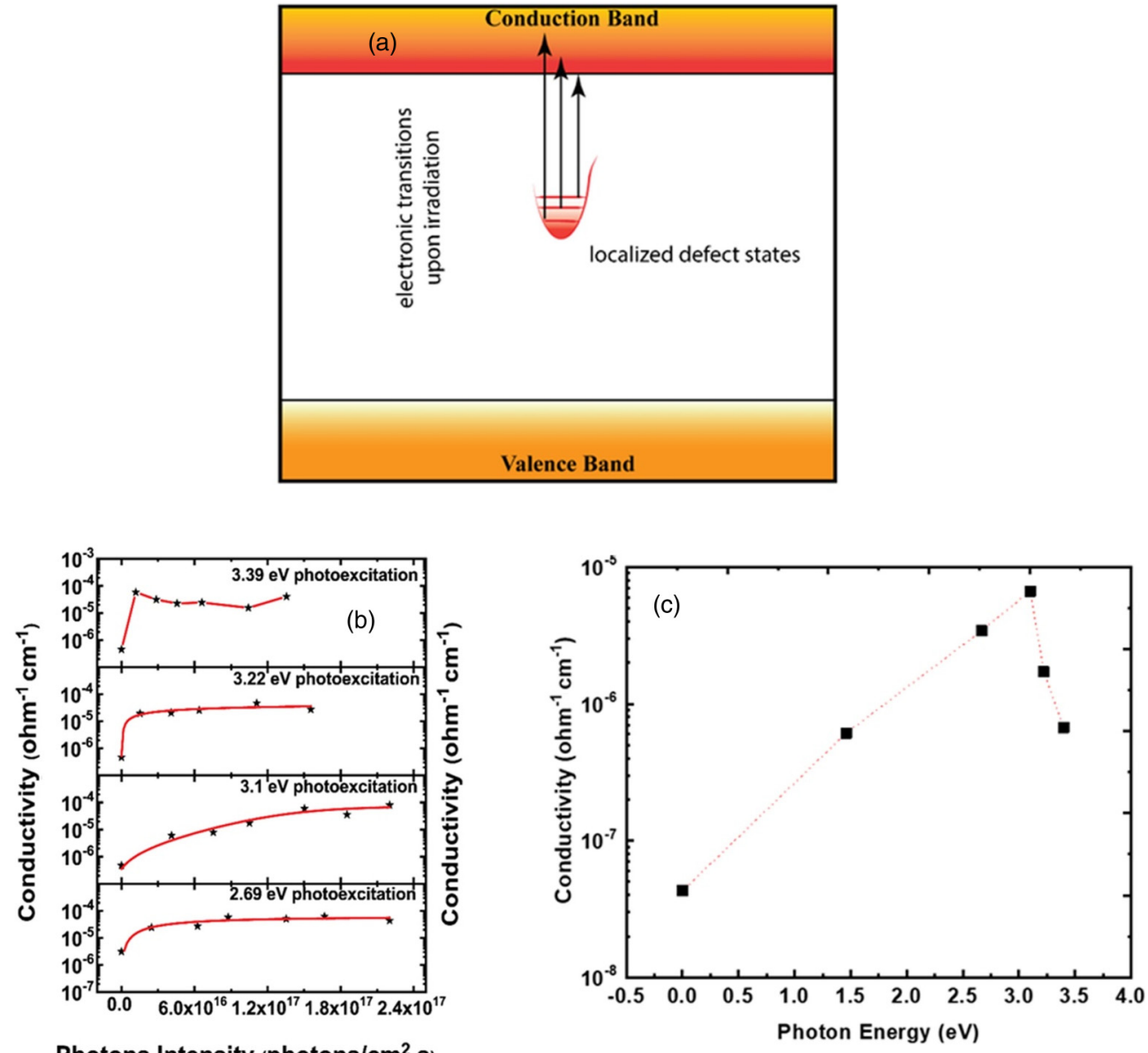

Photons Intensity (photons $/ \mathrm{cm}^{2} \mathbf{s}$ )

FIG. 1. Photoconductivity in undoped $\beta-\mathrm{Ga}_{2} \mathrm{O}_{3}$ bulk crystals measured at room temperature and 3 mtorr. (a) A schematic showing light-induced transition of electrons from localized states within the gap to the conduction band. (b) Dependence of photoconductivity on photoexcitation energy and intensity at room temperature. The initial dark conductivity before illuminating with 2.69 -eV photons was on the order of $10^{-6} \Omega^{-1} \mathrm{~cm}^{-1}$, which is one order of magnitude higher than the initial value in other measurements. (c) Dependence of photoconductivity on photon energy at room temperature.

$a=12.2140 \AA, b=3.03719 \AA, c=5.7819 \AA$, and $\beta=103.83^{\circ}$ [14]. It contains both octahedral and tetrahedral cation sites in equal numbers. As $\mathrm{Ga}_{2} \mathrm{O}_{3}$ has a wide band gap, it is an insulator at room temperature, but electron conduction has been reported when it is synthesized under reducing conditions [5]. This conductivity is often attributed to oxygen vacancies, though recent theoretical calculations showed that oxygen vacancies are deep states and cannot provide conduction electrons [6]. Electron conductivity can be realized in $\mathrm{Ga}_{2} \mathrm{O}_{3}$ by $\mathrm{Si}$ or Sn doping [10,11]. In fact, $\mathrm{Si}$ is a common impurity in $\mathrm{Ga}_{2} \mathrm{O}_{3}$ regardless of the growth method and is often a source of unintentional doping [26-28]. Achieving effective hole condition in $\mathrm{Ga}_{2} \mathrm{O}_{3}$ has been challenging; theoretical calculations show that the valance band is flat, indicating larger effective mass for holes, making $p$-type conductivity difficult [13]. However, we recently reported $p$-type conductivity with very high hole concentration and very low mobility [29].

\section{MATERIALS AND METHODS}

Czochralski (CZ) grown undoped, Fe-doped, and $\mathrm{Mg}$ doped bulk single crystals of $\mathrm{Ga}_{2} \mathrm{O}_{3}$ were obtained from
Synoptics Inc. The as-grown crystals were sliced into wafers of $1 \mathrm{~mm}$ thickness and the surface orientation of the wafers was 010. The electrical transport properties of the samples were measured in van der Pawn geometry using a Hall effect measurement system from MMR Technologies Inc. It is important to mention that serious efforts must be taken to ensure Ohmic contacts for $\mathrm{Ga}_{2} \mathrm{O}_{3}$ samples [30,31]. To achieve that, square samples of $5 \times 5 \mathrm{~mm}$ were properly cleaned, and indium contacts were soldered on the surface at the edge of the four corners of the samples. The In contacts were made using a solider heated to $700^{\circ} \mathrm{F}$. Then a linearity check was performed, confirming 4 Ohmic contacts. Light emitting diodes (LEDs) of various wavelengths $(365,385,400,460$, 650 , and $850 \mathrm{~nm}$ ) were used to provide photoexcitation of $3.39,3.22,3.1,2.69,1.9$, and $1.45 \mathrm{eV}$, respectively, and the photo-Hall measurements were carried out at room temperature. The intensity of photoexcitation was varied by changing the current passing through the LEDs. For the photo-Hall measurements, the Hall-effect chamber is customized with a transparent window for the illumination of the sample and a Joule Thompson refrigerator is used to keep the sample temperature constant, overcoming the heating effect caused by 
light illumination. The measurements were carried in vacuum of about a few mtorr. The refrigerator operates by running high pressure nitrogen gas through thin pipes. Because of the light induced heat, this setup is crucial for photoconductivity experiments to investigate the change in carrier concentration and conductivity due solely to photoexcitation, without the influence of thermal contributions. Temperature-dependent Hall effect measurements were performed on the permanent state conductive sample without illumination from 10 to $300 \mathrm{~K}$ using a cryostat with He compressor.

\section{THEORY/CALCULATION}

The structural properties of $\beta-\mathrm{Ga}_{2} \mathrm{O}_{3}$ were investigated by the density functional theory (DFT) formalism as implemented in VASP (Vienna Ab initio Simulation Package) [32]. The core electron behavior and the interaction between the valence electrons and the ion were described by the projector augmented wave method (PAW) [33]. The Perdew-BurkeErnzerhof (PBE) form of the generalized gradient approximation (GGA) was employed as the exchange-correlation functional to obtain the optimized ground state structure [34]. The Brillouin zone was sampled using $3 \times 3 \times 3$ and $7 \times 7 \times 7$ meshes of Monkhorst-Pack $k$ points for optimization and electronic structure calculations, respectively. The valence electrons were described by a plane waves basis set with a converged energy cutoff of $520 \mathrm{eV}$. A supercell of 160 atoms (32 formula unit) was considered in this calculation. The structure was optimized until the calculated HellmannFeynman forces were smaller than $0.0001 \mathrm{eV} \AA^{-1}$.

\section{RESULTS AND DISCUSSION}

$\mathrm{Ga}_{2} \mathrm{O}_{3}$ single crystals were illuminated by subband-gap light and the conductivity and carrier density were measured during illumination. Figure 1 illustrates the dependence of photoconductivity in undoped $\beta-\mathrm{Ga}_{2} \mathrm{O}_{3}$ single crystals on photoexcitation energy and intensity. In Fig. 1(b), the photoconductivity is plotted versus light intensity for photon energies of 3.39, 3.22, 3.1, $2.69 \mathrm{eV}$, revealing that the conductivity abruptly increases at low photon intensity and quickly saturates. In Fig. 1(c), the photoconductivity after reaching saturation is plotted as a function of photon energy. It is interesting to note that all subband-gap photoexcitations led to an increase in conductivity, even at low energies of $1.45 \mathrm{eV}$. The maximum photoconductivity occurs at $3.1 \mathrm{eV}$, which is much smaller than $4.5 / 4.9 \mathrm{eV}$, the band-gap energy of $\mathrm{Ga}_{2} \mathrm{O}_{3}$ [6]. The increase of conductivity with subband-gap photoexcitation can be explained due to the excitation of electrons from a localized state in the gap to the conduction band as shown in Fig. 1(a). In contrast to undoped $\mathrm{Ga}_{2} \mathrm{O}_{3}, \mathrm{Fe}-$ and $\mathrm{Mg}$-doped samples behave differently when exposed to light. Both Fe- and Mg-doped crystals showed decreased conductivity when exposed to 400- and 365-nm light (Fig. 2). This indicates that a light-induced change in the charge state of the $\mathrm{Fe}$ and $\mathrm{Mg}$ impurities, which act as compensating acceptors in $\mathrm{Ga}_{2} \mathrm{O}_{3}$, leads to a decrease in conductivity. Thus, the induced photoconductivity in Fig. 1 cannot be simply because of the change of the charge state of $\mathrm{Fe}$ and $\mathrm{Mg}$ impurities and the suppression of their acceptor role.

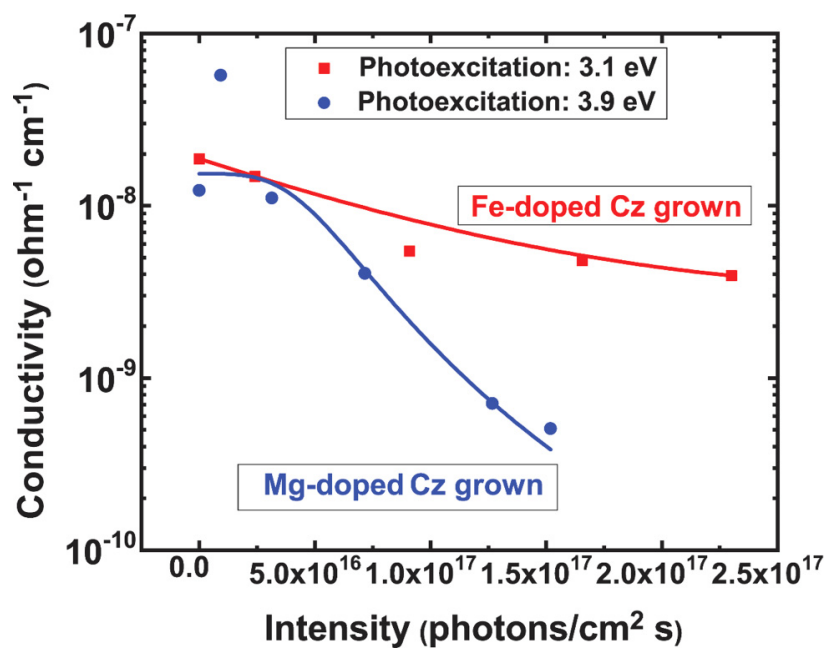

FIG. 2. Change in conductivity as a function of photon intensity in doped $\beta-\mathrm{Ga}_{2} \mathrm{O}_{3}$ bulk crystals: Fe-doped $\mathrm{Ga}_{2} \mathrm{O}_{3}$ and $\mathrm{Mg}$-doped $\mathrm{Ga}_{2} \mathrm{O}_{3}$. Contrary to undoped crystals, the conductivity here decreased by exposing the samples to light, illustrating that the induced photoconductivity of $\mathrm{Ga}_{2} \mathrm{O}_{3}$ in Fig. 1 is not due to light induced change in the charge state of compensating acceptors.

After turning off the photoexcitation, the undoped sample illuminated by $3.1 \mathrm{eV}$ shows persistent photoconductivity. To calculate the associated potential barrier that prevents the recapture of charge carriers by their centers after light is turned off and is thus the origin of the persistent photoconductivity $[3,35]$, the photoconductive sample was heated at various temperatures (from 300 to $390^{\circ} \mathrm{C}$ ) for $10 \mathrm{~min}$ at each temperature inside the Hall-effect chamber. After each heating, the sample was then cooled to room temperature and the electrical conductivity and carrier density were measured. The steps of the experimental procedure are illustrated in Fig. 3(a). Figure 3(b) shows how the conductivity and charge carrier density of the sample decay after heating to a certain temperature. We assume the carrier density $n$ changes with annealing temperature as $n=A e^{-E \text { th } / k T}$, where $k$ is the Boltzmann constant, $A$ is a constant, and $E_{\mathrm{th}}$ is the thermal energy barrier. Fitting of the data gives $E_{\mathrm{th}}$ of about $0.157 \pm 0.04 \mathrm{eV}$. In Fig. 3(c), we illustrate the process of electron pumping from the localized center to the conduction band where the center relaxes to a metastable state and the subsequent process of electron recapture through a barrier energy $E_{\text {th }}$. Surprisingly, subsequent prolonged and repeated photoexcitation lead to stable electron conduction that does not decay even after heating up to $400^{\circ} \mathrm{C}$ and does not follow anymore the trend and the mechanism illustrated in Fig. 3.

Figure 4 shows the electrical conductivity of the sample as a function of illumination time during exposure to $400 \mathrm{~nm}$ $(3.1 \mathrm{eV})$ excitation for a long period of time. The exposure was carried out inside the Hall chamber while holding the sample at room temperature and in vacuum of about a few mtorr. The conductivity abruptly increases at the beginning and remains almost constant after exposing the sample to light for $40 \mathrm{~min}$. The exposure was continued for $70 \mathrm{~h}$ and then turned off. Figure 5 presents the decay of the photoconductivity as a function of time after turning off the light. The decay 
(a)

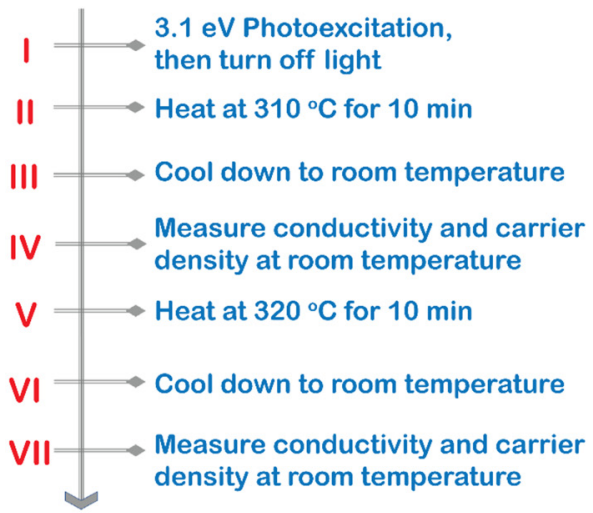

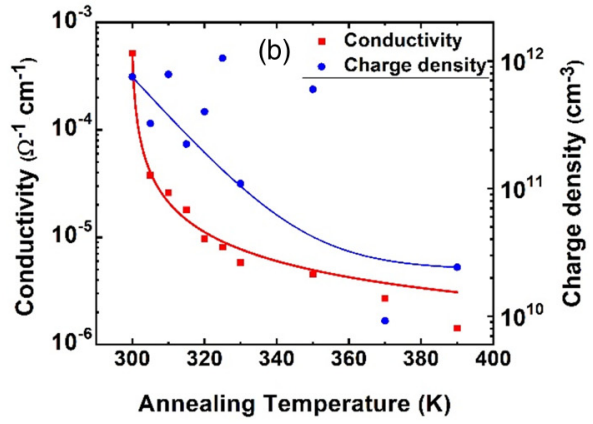

Annealing Temperature (K)

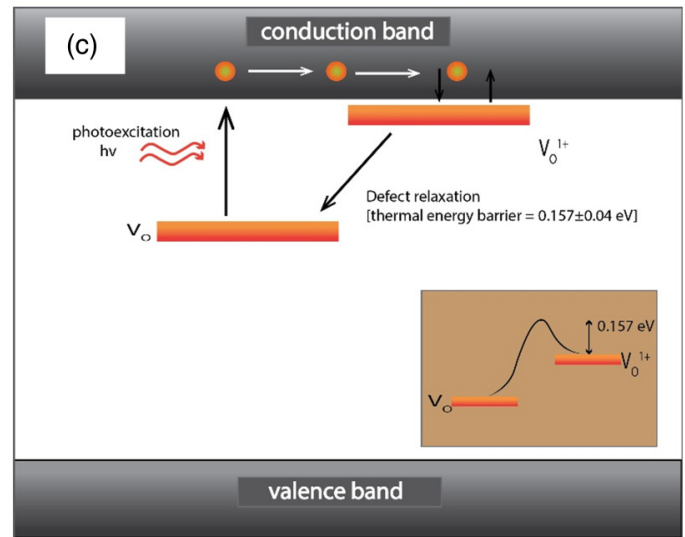

FIG. 3. Decrease of persistent photoconductivity and charge carrier density in undoped $\mathrm{Ga}_{2} \mathrm{O}_{3}$ by modest heating after removing photoexcitation and calculation of the thermal barrier energy for electron recapture by defect. (a) A diagram showing the experimental work flow. (b) Conductivity and carrier density versus heating temperature. This measurement shows the decrease of the induced conductivity by modest increase in temperature. (c) A schematic showing the excitation of an electron from the defect state to the conduction band and the thermal barrier energy for electron recapture.

of conductivity with time after turning the light off after 1 -h excitation is shown in Fig. 5(a). For photoexcitation of 3.39 and $3.22 \mathrm{eV}$, an immediate decline in electron conduction was observed after turning off the light. However, in the case of

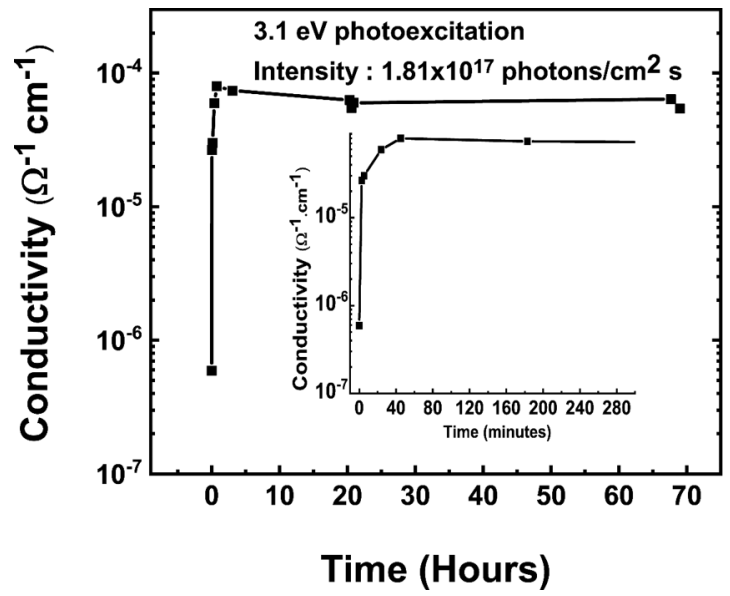

FIG. 4. Electrical conductivity in $\mathrm{Ga}_{2} \mathrm{O}_{3}$ as a function of illumination time (in situ measurements with light on). The inset highlights the increase in conductivity with illumination in the first several hours showing saturation around $40 \mathrm{~min}$.
3.1-eV excitation, the conductivity gradually decreased and attained the value of dark conductivity after $8 \mathrm{~min}$. This indicates the presence of more than one localized state in the band gap responsible for the photoconductivity and that the $3.1-\mathrm{eV}$ light most likely excites an electron from a center that exhibits a metastable state with longer decay time. Figure 5(b) (the blue curve) shows the decay of conductivity as a function of time after the 70-h exposure to $3.1-\mathrm{eV}$ excitation. After the decay shown in the blue curve, the sample was then re-exposed to $3.1-\mathrm{eV}$ photons again at higher intensity for $10 \mathrm{~min}$. The red curve in Fig. 5(b) represents the subsequent decay of conductivity after this short exposure. Each of the decay curves in Fig. 5(b) exhibits two time-decay constants, one relatively fast and one slow. The fast decay rate of conductivity was $0.4 \Omega^{-1} \mathrm{~cm}^{-1} /$ min after 1 -h excitation, $0.008 \Omega^{-1} \mathrm{~cm}^{-1} / \mathrm{min}$ after 70 -h excitation and $0.004 \Omega^{-1} \mathrm{~cm}^{-1} / \mathrm{min}$ after repeating photoexcitation with higher intensity. The dependence of photoconductivity decay on excitation time is unusual. However, these measurements clearly demonstrate the strong dependence of decay rate of conductivity on the energy, intensity, and time of photoexcitation. It is interesting that repeated exposure to light significantly impacts the decay of conductivity, leading to a more stable electron conduction. 

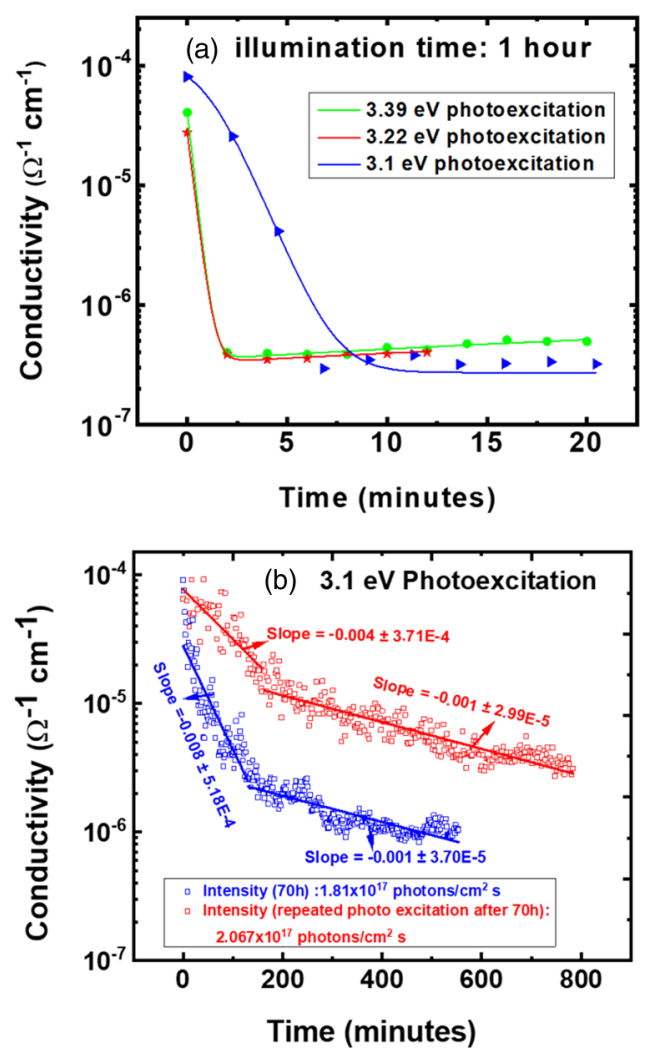

FIG. 5. Decay of photoconductivity in $\mathrm{Ga}_{2} \mathrm{O}_{3}$ sample as a function of time after turning off photoexcitation. (a) After exposing the sample to light with different photon energies for $1 \mathrm{~h}$. (b) After exposing the sample to $3.1-\mathrm{eV}$ photoexcitation. The blue curve represents the decay in conductivity after illuminating the sample for $70 \mathrm{~h}$. After the decay in conductivity after $70 \mathrm{~h}$ illumination and reaching the end of the blue curve, the sample was exposed again to higher photon intensity for $10 \mathrm{~min}$. The red curve shows the decay in conductivity after that. These results illustrate the dependence of the conductivity decay rate on the energy, intensity, and time of photoexcitation as well as on the repeated exposure to light.

To further investigate the conditions that cause the permanent transition from insulator to conductor state, an undoped $\mathrm{Ga}_{2} \mathrm{O}_{3}$ sample was exposed to light several times and the conductivity was monitored. Figure 6 summarizes the results. Initially the conductivity of the material was $1.08 \times 10^{-8} \Omega^{-1} \mathrm{~cm}^{-1}$. When exposed to photoexcitation of $3.1 \mathrm{eV}$, the conductivity promptly increased by almost two orders of magnitude but returned to essentially the original value after the light was turned off. However, by repeating photoexcitation and after prolonged exposure to light of $400 \mathrm{~nm}$ $(3.1 \mathrm{eV})$ of intensity of $2 \times 10^{17}$ photons $/ \mathrm{cm}^{2} \mathrm{~s}$ for about $90 \mathrm{~h}$, the conductivity was ultimately increased by nine orders of magnitude and remained constant at this value after turning off the light without decay, indicating a complete conversion from the insulator to conductor state. Annealing the sample at $400{ }^{\circ} \mathrm{C}$ for $1 \mathrm{~h}$ in dark did not remove or decrease the conductivity (Fig. 6). Note that indium contacts were completely removed from the sample before the annealing to ensure that In does not diffuse to the sample during annealing. Then new contacts were made after annealing and Hall effect measurements were done without exposing the sample to light; it is

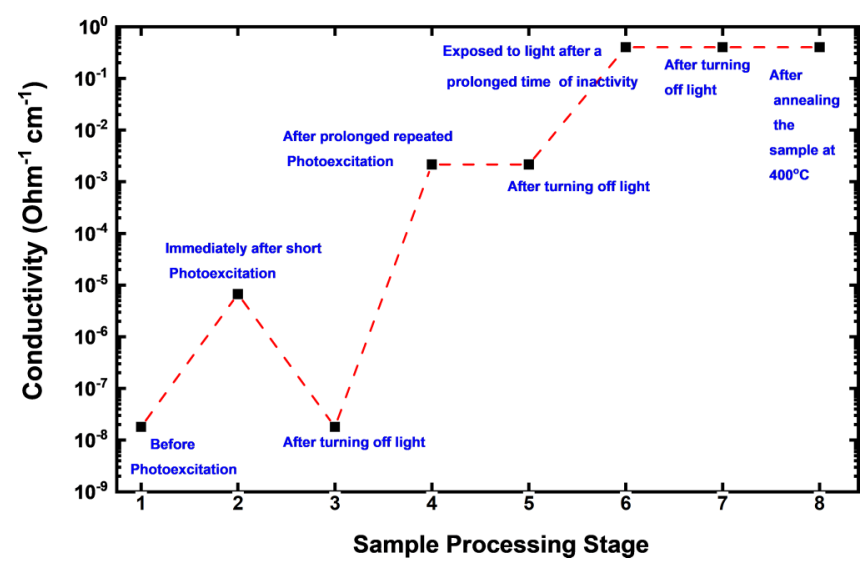

FIG. 6. Change in electron conduction in undoped $\beta-\mathrm{Ga}_{2} \mathrm{O}_{3}$ after exposing to photoexcitation several times. After repeated photoexcitation at $3.1 \mathrm{eV}$, the conductivity increased nine orders of magnitude from $10^{-8}$ to almost $1 \Omega^{-1} \mathrm{~cm}^{-1}$ and retained this conductivity without decay after turning off light, indicating a transition from insulator state to conductor state. Then, the conductivity was suppressed to about $10^{-6} \Omega^{-1} \mathrm{~cm}^{-1}$ by annealing the sample at $800^{\circ} \mathrm{C}$ in $\mathrm{O}_{2}$ for $2 \mathrm{~h}$ (not shown in the figure). The return to the initial conductivity of $10^{-8} \Omega^{-1} \mathrm{~cm}^{-1}$ may require annealing for longer time. After annealing at $800^{\circ} \mathrm{C}$, prolonged rephotoexcitation for $20 \mathrm{~h}$ did not increase the conductivity, on the contrary, it further decreased it illustrating that the sample lost its photoconductivity feature by high temperature anneal in $\mathrm{O}_{2}$.

important to emphasize that the conductivity was similar to its value before the anneal (Fig. 6). This procedure confirms that light-induced improvement of the contacts themselves does not contribute to the conductivity.

It is surprising that annealing at $400^{\circ} \mathrm{C}$ after turning off light did not bleach or decrease the photoconductivity. This clearly demonstrates the permanent transition from insulator state to conductor state. We emphasize that the $\mathrm{Ga}_{2} \mathrm{O}_{3}$ sample has transformed from highly insulating with $10^{-8} \Omega^{-1} \mathrm{~cm}^{-1}$ conductivity to a conductor with about $1 \Omega^{-1} \mathrm{~cm}^{-1}$ conductivity only by repeated exposure to subband-gap light for a period of time at room temperature, without any other treatment or thermal processing. This demonstrates an unusual phenomenon in this ultrawide band-gap material. Annealing at a much higher temperature of $800^{\circ} \mathrm{C}$ for $2 \mathrm{~h}$ in $\mathrm{O}_{2}$ flow followed by polishing the sample surface was necessary to revert the sample to an insulator with a conductivity of $7.69 \times 10^{-7} \Omega^{-1} \mathrm{~cm}^{-1}$. Additionally, after this annealing, we exposed the sample to photoexcitation for a long period of time; however, it did not show photoconductivity anymore. This means that this annealing also eliminated the photoconductivity feature of the sample. It should be noted that after each anneal, the sample surface was polished to facilitate soldering the indium contacts on the surface. Sample polishing also helps to remove any surface layer formed during annealing [36,37].

We exclude the effect of light on common impurities $\mathrm{Fe}$ and $\mathrm{Mg}$ acting as compensating acceptors in the sample as a possible source for the induced conductivity based on the results of Fig. 2. However, we consider in a later section the possible presence of other compensating acceptors. Again, 
the results in Fig. 2 illustrate that photoexcitation leads to a decrease in conductivity in $\mathrm{Mg}$ - and $\mathrm{Fe}$-doped $\mathrm{Ga}_{2} \mathrm{O}_{3}$. This means light induces a change in the charge state of $\mathrm{Mg}$ and Fe impurities, rendering them more effective compensating acceptors, hindering the conductivity. Thus, suppression of the role of compensating acceptors of impurities such as Fe or $\mathrm{Mg}$ by light cannot be responsible for the induced conductivity in undoped $\mathrm{Ga}_{2} \mathrm{O}_{3}$. However, point defects are thought to provide localized states in the band gap and may lead to persistent photoconductivity as illustrated in Figs. 1(a) and 3(d). The unusual permanent conversion from insulator to conductor observed here and the ability to eliminate this effect by annealing in $\mathrm{O}_{2}$ at high temperatures confirm the significant role of defects. Oxygen-annealing experiments completely eliminated the phenomenon and provided a strong evidence for the role of O-vacancy related defects. Based on that, we assume that the reported phenomenon in these undoped $\mathrm{Ga}_{2} \mathrm{O}_{3}$ crystals in this work is most likely related to the presence of large concentrations of oxygen vacancies. We propose this scenario: An oxygen vacancy $V_{O}$ in its neutral charge state forms a localized occupied deep state in the band gap and does not lead to conductivity. By exposing the sample to subband-gap light, electrons are pumped to the conduction band through two excitation steps producing a $V_{\mathrm{O}}{ }^{2+}$ state, which may provide shallow states. In fact, the temperature dependence of the induced conductivity and the electron density presented in Fig. 7 shows a freezeout region for the electrons (below $100 \mathrm{~K}$ ) indicating that these new states are still within the band gap.

To further understand the reason behind the permanent conversion from insulator to conductor and reveal the mechanism that prevented the electrons from returning to their center after turning off light, the change in the structural properties of $\beta-\mathrm{Ga}_{2} \mathrm{O}_{3}$ has been examined by first-principles electronic structure calculations. There are two different types of $\mathrm{Ga}$ sites present in the $\beta-\mathrm{Ga}_{2} \mathrm{O}_{3}$ crystal structure. The first is $\mathrm{Ga}$ coordinated by four oxygen (denoted as $\mathrm{Ga}_{1}$ ) while the second is $\mathrm{Ga}$ coordinated with six oxygen (denoted as $\mathrm{Ga}_{2}$ as shown in Fig. 8). The structure also possesses three inequivalent oxygen sites, two threefold coordinated $\mathrm{O}(\mathrm{I})$ and $\mathrm{O}(\mathrm{II})$ sites, and one fourfold coordinated O(III) site. Out of these three different sites, the $\mathrm{O}$ (II) site has the lowest formation energy for a neutral oxygen vacancy [33]. Therefore, in this study we have focused on O(II) type vacancy to evaluate the structural distortion due to the net charge on the defect. The calculations showed that in the presence of the neutral $\left(V_{\mathrm{O}}^{X}\right)$ and charged $\left(V_{\ddot{\mathrm{O}}}\right)$ oxygen vacancy, the Ga triangle (formed by the vacancy surrounded by three Ga atoms, denoted by the black dotted line in Fig. 8) contracts and expands respectively as the net charge on the vacancy is changed from neutral to positive. The average Ga-Ga bond length is 3.129 and $4.311 \AA$, respectively, for the neutral and charged cases. (For comparison, in the pristine (nondefective) structure, the average Ga-Ga bond length is $3.306 \AA$.) More specifically, in the case of $V_{\mathrm{O}}^{X}$, two $\mathrm{Ga}_{1}$ type atoms relax towards the vacancy site while the $\mathrm{Ga}_{2}$ type atom relaxes away from the vacancy. In contrast, in the $V_{\mathrm{O}}$ case, both the $\mathrm{Ga}_{1}$ and $\mathrm{Ga}_{2}$ type atoms relax away from the vacancy. The variation of the distance of $\mathrm{Ga}$ atoms from the vacancy with respect to the pristine structure is shown in Fig. 8. Therefore, inward and outward relaxation
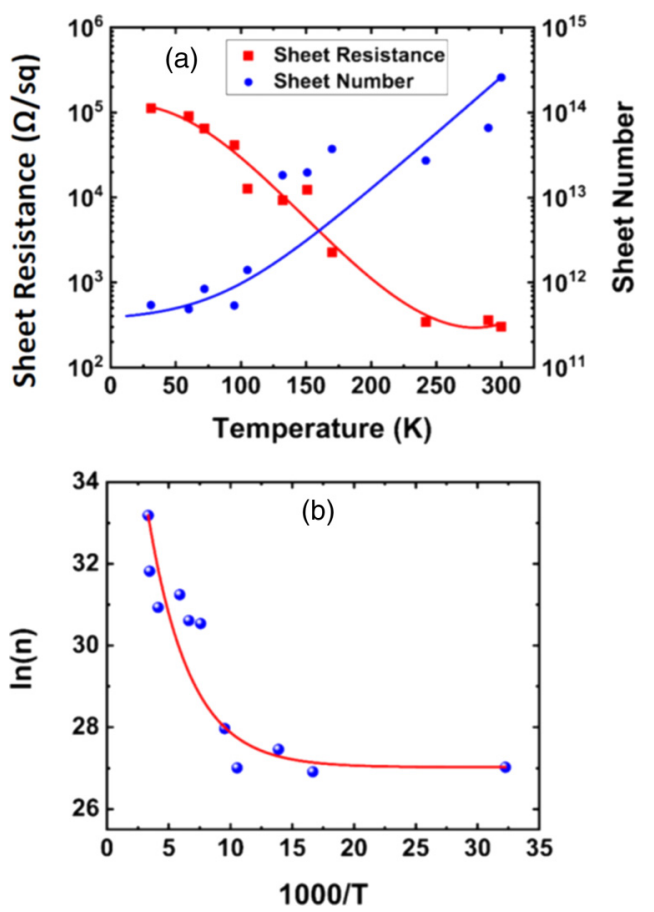

FIG. 7. Electronic conduction-temperature characteristics of the permanent state conductivity in $\mathrm{Ga}_{2} \mathrm{O}_{3}$ : (a) sheet resistance and sheet number (sheet carrier concentration: electrons $/ \mathrm{cm}^{2}$ ) as a function of temperature and (b) ln of sheet number as a function of inverse temperature. The measurements reveal a freeze out region for charge carriers indicating that the induced new states are shallow states within the band gap and not in the conduction band. The data points are scattered due to the noises associated with the long wires connecting the sample in the cryostat to the measuring unit. This is often noted in temperature dependent Hall effect measurements using a cryostat.

of the Ga triangle decreases (for $V_{\mathrm{O}}^{X}$ ) and increases (for $V_{\ddot{\mathrm{O}}}$ ) the average Ga-Ga bond length with respect to the pristine case.

To scrutinize the structural distortion around the vacancy, we analyzed the electron localization function (ELF) in the three systems. The ELF gives a direct spatial representation of the electron distribution, which is useful for examining bonding features. The local value of the ELF at a given position can be interpreted as the probability of finding an electron at that locality given the existence of neighboring electrons. The value of the ELF ranges from 0 to 1 . ELF values close to 1 suggest a region of space with high probability of finding electron localization, whereas a value of 0 corresponds to a region where either the electron is fully delocalized or does not reside. Finally, an ELF value close to $1 / 2$ implies that the region exhibits electron-gas-like behavior. Two-dimensional ELF contour plots of pristine $\mathrm{Ga}_{2} \mathrm{O}_{3}$ and $\mathrm{Ga}_{2} \mathrm{O}_{3}$ containing a neutral vacancy and a charged vacancy are shown in Fig. 9. The electron localization on $\mathrm{Ga}$ is lower than on $\mathrm{O}$, but there is strong overlap of electron localization. Redistribution of the electron localization probability is depicted for the case of the neutral $\mathrm{O}$ vacancy in Fig. 9(b), where a strong probability of finding an electron is observed between two $\mathrm{Ga}$ atoms near the vacancy site. This reduces the $\mathrm{Ga}_{1}-\mathrm{Ga}_{2}$ bond length 
(a)

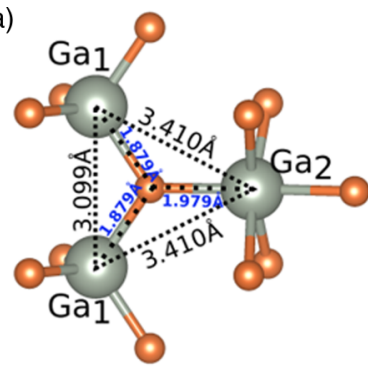

(b)

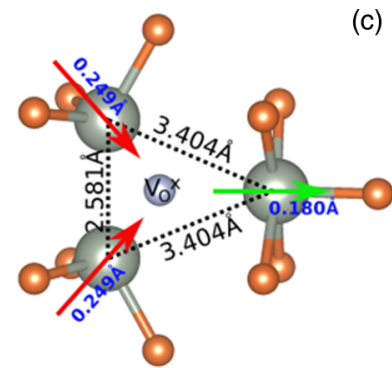

(c)

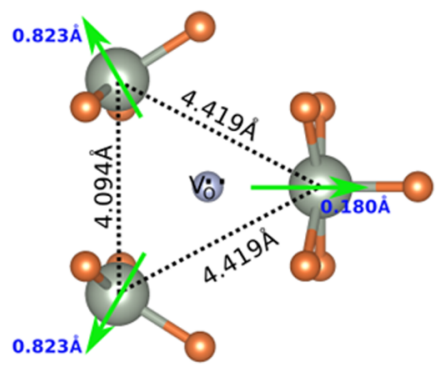

FIG. 8. Internal structural arrangements in $\beta-\mathrm{Ga}_{2} \mathrm{O}_{3}$ : (a) pristine structure, (b) with neutral oxygen vacancy, and (c) with doubly charged oxygen vacancy. Variation in the distance of Ga atoms from the vacancy is also shown. Red and green arrows with indicated values show how much $\mathrm{Ga}$ atoms move towards or away from the vacancy, respectively. Grey and orange balls represent $\mathrm{Ga}$ and $\mathrm{O}$ atoms, respectively.

and pushes $\mathrm{Ga}_{1}$ towards the defect site. The $\mathrm{Ga}_{2}$ atom also interacts slightly with this localized charged state, but it has a much stronger interaction with the neighboring $\mathrm{O}$, which pulls $\mathrm{Ga}_{2}$ away from the defect site. In the charged vacancy case [Fig. 9(c)], an ELF quite similar to the pristine case is observed except around the defect site. Moreover, the ELF does not exhibit the localized charge state between two $\mathrm{Ga}_{1}$ atoms. Rather, the $\mathrm{Ga}_{1}$ atoms are displaced from their original positions and move away from the defect site to form a new $\mathrm{Ga}_{1}-\mathrm{O}$ bond. However, the ELF of the $\mathrm{Ga}_{2}$ atom is similar to the neutral vacancy case. This results in the massive expansion of the $\mathrm{Ga}$ triangle and corresponding increase in $\mathrm{Ga}_{1}-\mathrm{Ga}_{1}$, $\mathrm{Ga}_{1}-\mathrm{Ga}_{2}$ bond lengths.

The total and partial electronic density of states (DOS) are plotted in Fig. 10 to provide insight about the impact of the structural relaxation of neutral and charged vacancy on the electronic states within the material. The DOS of the pristine structure is also shown for comparison. It is observed in Fig. 10 that the presence of the neutral vacancy introduces an occupied localized state just above the valence band due to the internal inward relaxation shown in Fig. 8(b). On the other hand, in the case of the charged oxygen vacancy, the outward relaxation of the Ga triangle shifts the defect states to higher energy, closer to the conduction band minimum region (in the DOS in Fig. 10, these overlap with the conduction band in a way that makes it difficult to discern by eye). Those states are now unoccupied.
These calculations reveal that changing the charge state of the vacancy — as takes place during photoexcitation-leads to a strong structural relaxation and, as would be expected, a change in the defect electronic states in the band gap. They confirm the experimental scenario that the vacancy structure changes when it is excited. Once the vacancy charge state changes, not only is the defect state emptied, but the state shifts significantly towards the conduction band, leading to a situation in which there is no energetic driving force for the excited electrons to re-encounter the vacancy. This is different from common persistent photoconductivity and what is presented in Fig. 3, where charge carriers can return to their initial states by overcoming the induced potential barrier. It should be emphasized that short exposure to light induced common persistent photoconductivity (Fig. 3), but subsequent longer exposure to light led to a complete nonreversal transition from insulator to conductor.

While our computations showed that changing the charge state of $\mathrm{O}$ vacancies by light can lead to a significant lattice relaxation and massive structural changes in the lattice leading to the persistent conductivity, it should be also noted that light-induced changes in compensating acceptors such as $\mathrm{Ga}$ vacancy-related defects may play a role in the observed phenomenon. Ga vacancies and related defects are known to act as compensating acceptors in $\mathrm{Ga}_{2} \mathrm{O}_{3}[4,10,38]$. Regardless of the microscopic origin of the defect centers in the crystals, the observed phenomenon of permanent transition from insulator (a)

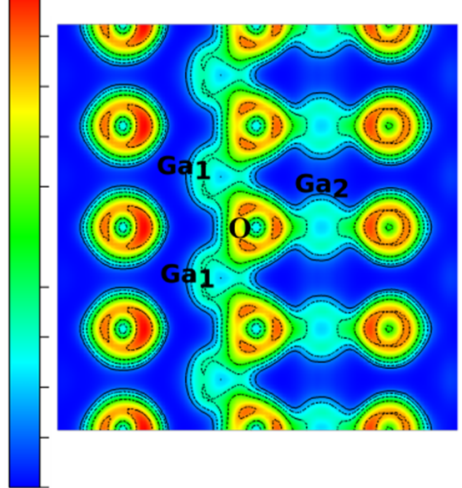

(b)

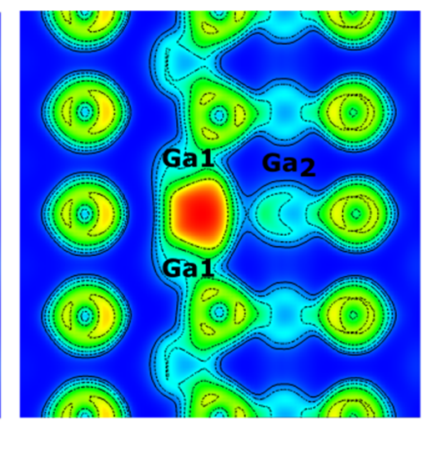

(c)

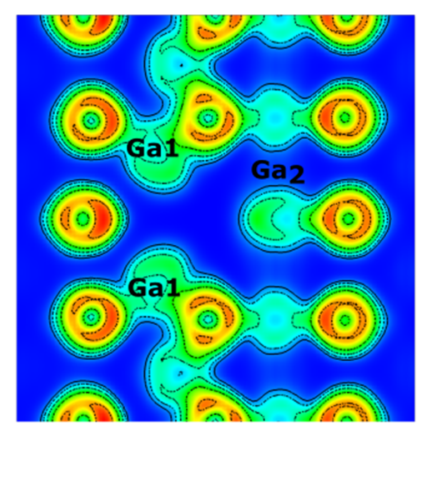

FIG. 9. Two-dimensional contour plots of the electron localization function (ELF) for (a) pristine $\mathrm{Ga}_{2} \mathrm{O}_{3}$, (b) $\mathrm{Ga}_{2} \mathrm{O}_{3}$ containing a neutral oxygen vacancy, and (c) $\mathrm{Ga}_{2} \mathrm{O}_{3}$ containing a 2+ charged oxygen vacancy. Blue, green, and red correspond to ELF values of 0 , 0.5, and 1, respectively. 

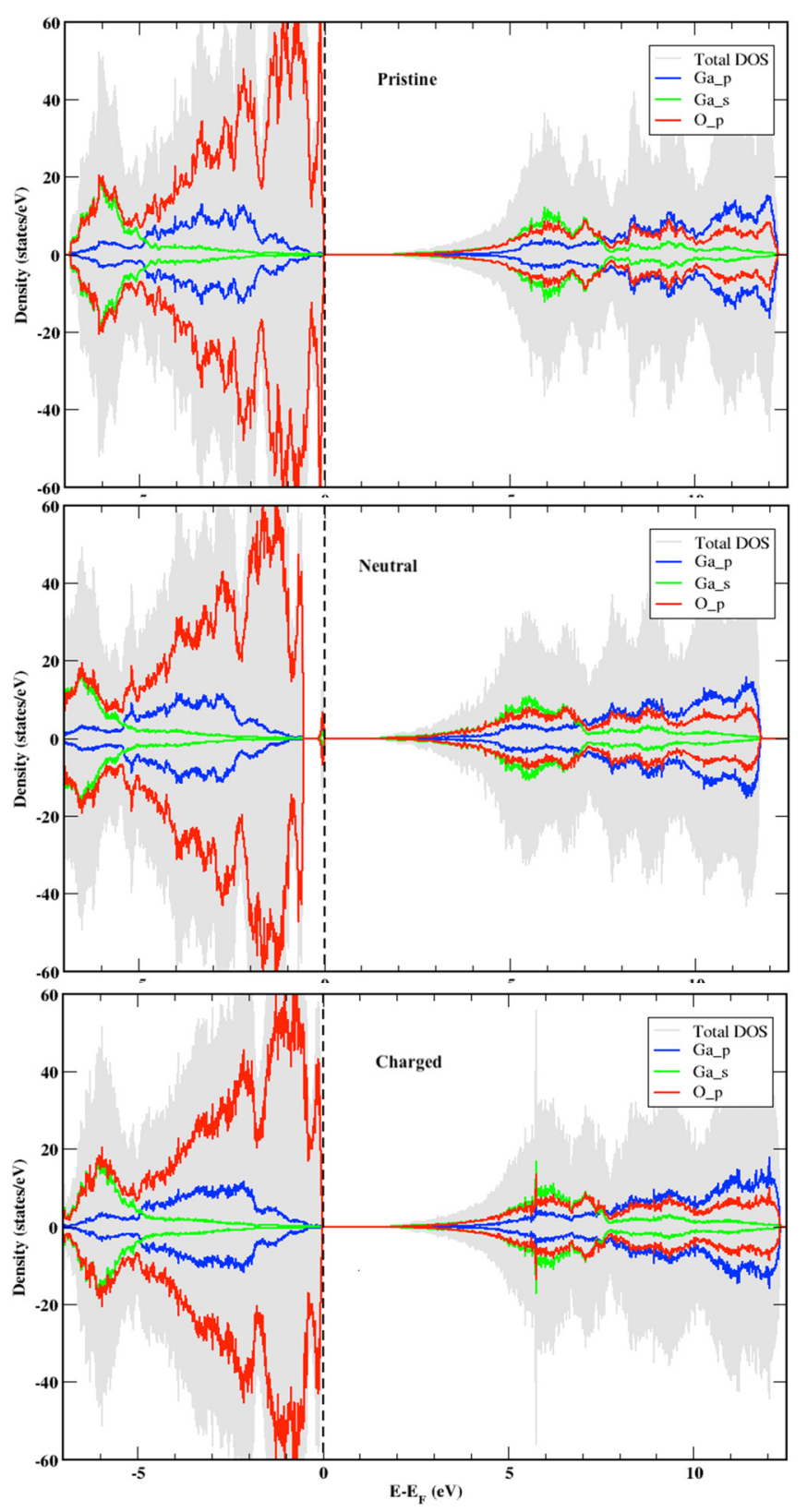

FIG. 10. Total (gray) and partial (lines) electronic density of states (DOS) of (a) pristine $\mathrm{Ga}_{2} \mathrm{O}_{3}$ and $\mathrm{Ga}_{2} \mathrm{O}_{3}$ with (b) a neutral and (c) charged oxygen vacancy. The $s$ and $p$ partial DOS for Ga and the $p$ partial DOS for oxygen are represented by the green, blue, and red lines, respectively. The zero of energy is referenced against the highest occupied state in the system, referred to here as the Fermi energy $E_{\mathrm{F}}$. Positive and negative DOS correspond to spin up and spin down components, respectively.

to conductor by only repeating exposure to subband-gap light for short period of times at room temperature without any further treatment is quite unusual and intriguing. It reveals that, in ultrawide band-gap materials, light can induce a permanent structural change in the lattice-either contraction or expansion in the vicinity of the defect center-modifying the electronic states and material properties. The finding of this work should inspire new inquiries on the possible effect of light on the electronic states of wide band-gap materials and subsequently their electrical, optical, and magnetic properties. This can have massive impact on understanding the functionality and stability of such wide band-gap materials in devices.

Finally, it is worth contrasting the behavior here to the mechanism described in Ref. [2]. Fundamentally, the mechanism is very similar. The main difference relates to how able electrons can return to the anion vacancy to eliminate the persistent conductivity. In the mechanism in Ref. [2], once the light is removed, the electrons are able to return to the vacancy; as a consequence, the free carriers vanish. Here, the free charge carriers persist, as the electrons cannot recombine with the vacancy, and thus so does the conductivity. We speculate that the difference arises to a larger structural relaxation in $\mathrm{Ga}_{2} \mathrm{O}_{3}$ than in $\mathrm{ZnO}$, leading to a longer time scale (equivalently, larger barrier) for electron-vacancy recombination. However, to prove this, we would need to calculate the excited electron relaxation time for both materials, which is beyond the scope of the present work.

\section{CONCLUSIONS}

We have revealed a mechanism for insulator conductor transition through the redistribution of electron localization in the lattice induced by altering the charge states of defect centers and the subsequent drastic lattice distortion and large shift in the density of states. Experiments demonstrated that subband-gap light illumination of undoped $\mathrm{Ga}_{2} \mathrm{O}_{3}$ for limited times leads to a permanent transition from a highly insulating state to a conductive state that cannot be reversed, an unusual and surprising phenomenon with vast implications on both the properties and potential applications of the material. Theoretical findings revealed that this transition is due to lattice distortion induced by changes in the charge state of defects and subsequent modifications in the electronic states. We propose that such a transition may take place in other wide band-gap materials, strongly impacting their properties and applications. We also propose that the dependence of the decay of conductivity on the photoexcitation time and intensity revealed in this work may open up a frontier to tuning material properties and developing devices that can be controlled by light. By limiting the excitation time or decreasing the photon intensity, conductivity can be generated and erased providing opportuning for optical memory uses. Further, long time excitation can be used to develop an $n$-type semiconductor for electronics.

All data needed to evaluate the conclusions in the paper are present in the paper. Additional data are available from the authors upon request: for experimental work, contact faselim@bgsu.edu; for computational work, contact blas@lanl.gov.

\section{ACKNOWLEDGMENTS}

The experimental work was supported by the National Science Foundation (NSF) under Grant No. DMR-2005064. The theory and computational work were supported as part of FUTURE, an Energy Frontier Research Center funded by the U.S. Department of Energy, Office of Science, Basic Energy Sciences. Los Alamos National Laboratory is operated by 
Triad National Security, LLC, for the National Nuclear Security Administration of U.S. Department of Energy (Contract No. 89233218CNA000001).

F.A.S. developed the idea and interpreted the results, D.R., S.A., M.I., P.S., P.D., and N.A. carried out the annealing and conductivity experiments, M.B., M.O.L., and A.W. contributed to the interpretation of the origin of the defect centers, A.B. and B.P.U. performed DFT calculations and analysis, and F.A.S. and B.P.U. wrote the manuscript.

The authors declare no competing financial interest.
[1] R. H. Bube and S. A. Rice, Photoconductivity of solids, Phys. Today 14(5), 54 (1961).

[2] S. Lany and A. Zunger, Anion vacancies as a source of persistent photoconductivity in II-VI and chalcopyrite semiconductors, Phys. Rev. B 72, 035215 (2005).

[3] M. C. Tarun, F. A. Selim, and M. D. McCluskey, Persistent Photoconductivity in Strontium Titanate, Phys. Rev. Lett. 111, 187403 (2013).

[4] P. Saadatkia, S. Agarwal, A. Hernandez, E. Reed, I. D. Brackenbury, C. L. Codding, M. O. Liedke, M. Butterling, A. Wagner, and F. A. Selim, Point and extended defects in heteroepitaxial $\beta-\mathrm{Ga}_{2} \mathrm{O}_{3}$ films, Phys. Rev. Mater. 4, 104602 (2020).

[5] N. Ueda, H. Hosono, R. Waseda, and H. Kawazoe, Synthesis and control of conductivity of ultraviolet transmitting $\beta-\mathrm{Ga}_{2} \mathrm{O}_{3}$ single crystals, Appl. Phys. Lett. 70, 3561 (1997).

[6] J. B. Varley, J. R. Weber, A. Janotti, and C. G. Van de Walle, Oxygen vacancies and donor impurities in $\beta-\mathrm{Ga}_{2} \mathrm{O}_{3}$, Appl. Phys. Lett. 97, 142106 (2010).

[7] S. I. Stepanov, V. I. Nikolaev, V. E. Bougrov, and A. E. Romanov, Gallium oxide: Properties and applications A review, Rev. Adv. Mater. Sci. 44, 63 (2016).

[8] M. M. Islam, D. Rana, A. Hernandez, M. Haseman, and F. A. Selim, Study of trap levels in $\beta-\mathrm{Ga}_{2} \mathrm{O}_{3}$ by thermoluminescence spectroscopy, J. Appl. Phys. 125, 055701 (2019).

[9] J. Huso, M. D. McCluskey, Y. Yu, M. M. Islam, and F. Selim, Localized UV emitters on the surface of $\beta-\mathrm{Ga}_{2} \mathrm{O}_{3}$, Sci. Rep. 10, 21022 (2020).

[10] M. M. Islam, N. Adhikari, A. Hernandez, A. Janover, S. Novak, S. Agarwal, C. L. Codding, M. Snure, M. Huang, and F. A. Selim, Direct measurement of the density and energy level of compensating acceptors and their impact on the conductivity of n-type $\mathrm{Ga}_{2} \mathrm{O}_{3}$ films, J. Appl. Phys. 127, 145701 (2020).

[11] M. Higashiwaki, K. Sasaki, H. Murakami, Y. Kumagai, A. Koukitu, A. Kuramata, T. Masui, and S. Yamakoshi, Recent progress in $\mathrm{Ga}_{2} \mathrm{O}_{3}$ power devices, Semicond. Sci. Technol. 31, 034001 (2016).

[12] R. Roy, V. G. Hill, and E. F. Osborn, Polymorphism of $\mathrm{Ga}_{2} \mathrm{O}_{3}$ and the system $\mathrm{Ga}_{2} \mathrm{O}_{3}-\mathrm{H}_{2} \mathrm{O}$, J. Am. Chem. Soc. 74, 719 (1952).

[13] H. He, M. A. Blanco, and R. Pandey, Electronic and thermodynamic properties of $\beta-\mathrm{Ga}_{2} \mathrm{O}_{3}$, Appl. Phys. Lett. 88, 261904 (2006).

[14] J. A. Kohn, G. Katz, and J. D. Broder, Characterization of $\beta$ $\mathrm{Ga}_{2} \mathrm{O}_{3}$ and its Alumina Isomorph, $\theta-\mathrm{Al}_{2} \mathrm{O}_{3}$, Am. Mineral.: J. Earth Planet. Mater. 42, 398 (1957) .

[15] Z. Guo, A. Verma, X. Wu, F. Sun, A. Hickman, T. Masui, A. Kuramata, M. Higashiwaki, D. Jena, and T. Luo, Anisotropic thermal conductivity in single crystal $\beta$-gallium oxide, Appl. Phys. Lett. 106, 111909 (2015).

[16] N. Suzuki, S. Ohira, M. Tanaka, T. Sugawara, K. Nakajima, and T. Shishido, Fabrication and characterization of transpar- ent conductive $\mathrm{Sn}$-doped $\beta-\mathrm{Ga}_{2} \mathrm{O}_{3}$ single crystal, Phys. Status Solidi C 4, 2310 (2007).

[17] H. Aida, K. Nishiguchi, H. Takeda, N. Aota, K. Sunakawa, and Y. Yaguchi, Growth of $\beta-\mathrm{Ga}_{2} \mathrm{O}_{3}$ single crystals by the edgedefined, film fed growth method, Jpn. J. Appl. Phys. 47, 8506 (2008).

[18] K. Sasaki, A. Kuramata, T. Masui, E. G. Villora, K. Shimamura, and $\mathrm{S}$. Yamakoshi, Device-quality $\beta-\mathrm{Ga}_{2} \mathrm{O}_{3}$ epitaxial films fabricated by ozone molecular beam epitaxy, Appl. Phys. Express 5, 035502 (2012).

[19] Y. Lv, J. Ma, W. Mi, C. Luan, Z. Zhu, and H. Xiao, Characterization of $\beta-\mathrm{Ga}_{2} \mathrm{O}_{3}$ thin films on sapphire (0001) using metal-organic chemical vapor deposition technique, Vacuum 86, 1850 (2012).

[20] M. Fleischer, W. Hanrieder, and H. Meixner, Stability of semiconducting gallium oxide thin films, Thin Solid Films 190, 93 (1990).

[21] J. Zhang, J. Shi, D.-C. Qi, L. Chen, and K. H. L. Zhang, Recent progress on the electronic structure, defect, and doping properties of $\mathrm{Ga}_{2} \mathrm{O}_{3}$, APL Mater. 8, 020906 (2020).

[22] A. Hernandez, M. M. Islam, P. Saddatkia, C. Codding, P. Dulal, S. Agarwal, A. Janover et al., MOCVD growth and characterization of conductive homoepitaxial Si-doped $\mathrm{Ga}_{2} \mathrm{O}_{3}$, Results Phys. 25, 104167 (2021).

[23] M. Orita, H. Hiramatsu, H. Ohta, M. Hirano, and H. Hosono, Preparation of highly conductive, deep ultraviolet transparent $\beta-\mathrm{Ga}_{2} \mathrm{O}_{3}$ thin film at low deposition temperatures, Thin Solid Films 411, 134 (2002).

[24] K. Sasaki, M. Higashiwaki, A. Kuramata, T. Masui, and S. Yamakoshi, MBE grown $\mathrm{Ga}_{2} \mathrm{O}_{3}$ and its power device applications, J. Cryst. Growth 378, 591 (2013).

[25] M. R. Lorenz, J. F. Woods, and R. J. Gambino, Some electrical properties of the semiconductor $\beta-\mathrm{Ga}_{2} \mathrm{O}_{3}$, J. Phys. Chem. Solids 28, 403 (1967).

[26] A. Kuramata, K. Koshi, S. Watanabe, Y. Yamaoka, T. Masui, and $\mathrm{S}$. Yamakoshi, High-quality $\beta-\mathrm{Ga}_{2} \mathrm{O}_{3}$ single crystals grown by edge-defined film-fed growth, Jpn. J. Appl. Phys. 55, 1202A2 (2016).

[27] K. Irmscher, Z. Galazka, M. Pietsch, R. Uecker, and R. Fornari, Electrical properties of $\beta-\mathrm{Ga}_{2} \mathrm{O}_{3}$ single crystals grown by the Czochralski method, J. Appl. Phys. 110, 063720 (2011).

[28] Z. Galazka, R. Uecker, K. Irmscher, M. Albrecht, D. Klimm, M. Pietsch, M. Brützam, R. Bertram, S. Ganschow, and R. Fornari, Czochralski growth and characterization of $\beta-\mathrm{Ga}_{2} \mathrm{O}_{3}$ single crystals, Cryst. Res. Technol. 45, 1229 (2010).

[29] M. M. Islam, M. O. Liedke, D. Winarski, B. Maik, A. Wagner, P. Hosemann, Y. Wang, U. Blas, and F. A. Selim, Chemical manipulation of hydrogen induced high p-type and n-type conductivity in $\mathrm{Ga}_{2} \mathrm{O}_{3}$, Sci. Rep. 10, 6134 (2020).

[30] S. J. Pearton, J. Yang, P. H. Cary IV, F. Ren, J. Kim, M. J. Tadjer, and M. A. Mastro, A review of $\mathrm{Ga}_{2} \mathrm{O}_{3}$ materials, processing, and devices, Appl. Phys. Rev. 5, 011301 (2018). 
[31] M.-H. Lee and R. L. Peterson, Accelerated Aging Stability of $\beta$ - $\mathrm{Ga}_{2} \mathrm{O}_{3}-$ Titanium/Gold Ohmic Interfaces, ACS Appl. Mater. Interfaces 12, 46277 (2020).

[32] G. Kresse and J. Furthmüller, Efficient iterative schemes for ab initio total-energy calculations using a plane-wave basis set, Phys. Rev. B 54, 11169 (1996).

[33] P. E. Blöchl, Projector augmented-wave method, Phys. Rev. B 50, 17953 (1994).

[34] J. P. Perdew, K. Burke, and M. Ernzerhof, Generalized Gradient Approximation Made Simple, Phys. Rev. Lett. 77, 3865 (1996).

[35] A. Tebano, E. Fabbri, D. Pergolesi, G. Balestrino, and E. Traversa, Room-temperature giant persistent photoconductivity in $\mathrm{SrTiO}_{3} / \mathrm{LaAlO}_{3}$ heterostructures, ACS Nano 6, 1278 (2012).
[36] T. Oshima, K. Kaminaga, A. Mukai, K. Sasaki, T. Masui, A. Kuramata, S. Yamakoshi, S. Fujita, and A. Ohtomo, Formation of semi-insulating layers on semiconducting $\beta-\mathrm{Ga}_{2} \mathrm{O}_{3}$ single crystals by thermal oxidation, Jpn. J. Appl. Phys. 52, 051101 (2013).

[37] Z. Galazka, K. Irmscher, R. Uecker, R. Bertram, M. Pietsch, A. Kwasniewski, M. Naumann et al., On the bulk $\beta-\mathrm{Ga}_{2} \mathrm{O}_{3}$ single crystals grown by the Czochralski method, J. Cryst. Growth 404, 184 (2014).

[38] S. K. Swain, M. H. Weber, J. Jesenovec, M. Saleh, K. G. Lynn, and J. S. McCloy, Compensation of Shallow Donors by Gallium Vacancies in Monoclinic $\beta-\mathrm{Ga}_{2} \mathrm{O}_{3}$, Phys. Rev. Appl. 15, 054010 (2021). 